\title{
Model-driven estimation of operators' workload for usage centred design of interactive systems
}

Conference Paper · March 2015

DOI: 10.1109/COGSIMA.2015.7108176

CITATION

1

\section{2 authors:}

Dhouha Kbaier Ben Ismai University of Wolverhampton 27 PUBLICATIONS $\mathbf{5 0}$ CITATIONS

SEE PROFILE
READS

87

Olivier Grisvard

Institut Mines-Télécom

16 PUBLICATIONS 10 CITATIONS

SEE PROFILE

Some of the authors of this publication are also working on these related projects:

MEDUSA View project

MASDOC View project 


\title{
Model-driven estimation of operators' workload for usage centred design of interactive systems
}

\author{
Dhouha Kbaier Ben Ismail ${ }^{1,2}$ and Olivier Grivard ${ }^{1,2,3}$ \\ ${ }^{1}$ Institut Mines-Télécom - Télécom Bretagne - UMR CNRS 3192 Lab-STICC \\ ${ }^{2}$ Université Européenne de Bretagne \\ ${ }^{3}$ Thales Systèmes Aéroportés \\ \{dhouha.kbaier, olivier.grisvard $\} @$ telecom-bretagne.eu
}

\begin{abstract}
The measurement of the operators' workload is an important aspect of usage-oriented design of professional systems. In domains such as avionics, air traffic management or mission systems, being able to quantify the operators' workload under stress, and in potentially demanding physical and mental conditions, is mandatory to anticipate overload and prevent human errors. Current approaches to workload estimation rely mainly on experimentation in simulation as an approach that has proven its efficiency for the identification of bad system and/or user interface design. Even if one cannot expect to totally avoid experimenting, given the complexity of the issue of workload computation, a priori estimation of workload might be an interesting tool to pre-validate a design in order to save some time in the experimentation phase and facilitate the analysis of overload situations that appear during experimentation. Various approaches to the a priori measurement of workload have been proposed: performance-based, physiological and subjective measures. Although performance and physiological measures of workload may be more precise, subjective measures are more practical, easier and less costly to use. For these reasons, they have been applied to many complex domains. The experience, the skills and the level of training of the operator have been identified in the literature as being important human factors. Nevertheless, these parameters have not been deeply analyzed in the context of workload estimation. In this paper, we develop a predictive workload model based on the analysis of the tasks assigned to a human operator. We propose to use mental representations of tasks, human actors, human roles, knowledge and abilities. We then propose to estimate the operator's workload with reference to his experience and training, the load over time and the task complexity. Our approach is illustrated on an airborne maritime surveillance use-case, in the context of the French Medusa project.
\end{abstract}

Keywords-workload; model-driven engineering; humanmachine interface; human-computer interaction; mental representation; tasks; roles; knowledge; abilities; maritime surveillance.

\section{INTRODUCTION}

Workload measurement has been applied to a number of military and industrial problems. There are three major types of workload measure: performance-based, physiological and subjective. First, performance-based measures can be subdivided into primary-task and secondary-task measures. Primary-task measures provide a direct indication of performance on the task under consideration. However, performance on the primary task may be insensitive to workload change if operators compensate by increased effort.
The secondary task is an additional measure to the primary task. "The basic idea of a secondary task is that it measures the difference between the 'mental capacity' consumed by the main task, and the total available capacity" (Mulder, 1979) [1]. The major problem that may occur when secondary tasks are used to measure workload is that they may disrupt primary task performance (Colle \& Reid, 1999; Sirevaag et al., 1993) [2, 3]. For example, a verbal secondary task may not interfere with a spatial primary task, even if the primary task is very demanding. Physiological measures are based on the assumption that workload will induce physical changes. These changes are measured in cardiac activity, brain activity, breath activity, speech measures, and eye activity. An operator who is overloaded may experience changes such as increase in heartbeat rate and skin conductance. Often, a large volume of data is collected, requiring unfortunately sophisticated analysis. Finally, subjective measures are used to reflect the amount of information used in working memory (Yeh \& Wickens, 1988) [4]. A simplistic, but realistic, way to look at workload measurement is that "if the person feels loaded and effortful, he is loaded and effortful, whatever the behavioral and performance measure show" (Johannsen et al., 1979) [5]. Thus, subjective measurement is based on the use of scales to measure the amount of workload a person is feeling.

Although physiological measures of workload may be more precise, subjective measures are more practical. Furthermore, subjective tests are flexible for different people with different capabilities. "Because subjective ratings take into account individual differences in ability, state, and attitude differences that may be obscured in objective measures of performance until breakdown makes them obvious - they are valuable because of, not despite, their subjectivity" (Muckler \& Seven, 1992) [6]. Even though subjective and objective measures of workload are very different, it has been shown that subjective measures correlate with physiological measures of workload such as heartbeat rate variability (Tattersall \& Foord, 1996) [7]. Adding to that, an increasing number of studies have found operator ratings to be a more direct indicator of workload than physical measures. Subjective measures are considered to be the least intrusive, most flexible, most convenient, least time consuming, and least expensive form of evaluating workload (Yeh \& Wickens, 1988) [4]. A variety of subjective measures have been developed and applied in many studies, particularly those of the flight deck. One of the most widely used scales is the NASA Task Load Index (Hart and 
Staveland, 1988) [8]. The NASA-TLX has been implemented in many aviation studies since it is considered to be a good multidimensional scale. It uses six dimensions to assess workload: mental demand, physical demand, temporal demand, performance, effort and frustration. The six subscales can be divided into three groups. It fact mental, physical and temporal demand are characteristics of the task; performance and effort are behavioral characteristics and frustration is assumed to be individual characteristic.

In this paper, we present a new method to estimate workload that calls back the three important factors aforementioned: characteristics of the task, behavioral characteristics and individual characteristics. In fact, we argue that workload can be inferred from analysis of tasks required of human operator. However, individual differences must be taken into account. For example, a novice and expert will obviously experience different levels of workload when performing the same task. For this reason, we explore the following important parameters: task complexity, time load, experience, knowledge and abilities compared to task requirements. Since 'frustration' and 'physical demand' have only shown a small relevance for workload (Pfendler and Widdel, 1988; Sepehr, 1988; Veltman and Gaillard, 1993) [912], these subscales of the NASA-TLX have not been included in our analysis. The paper is organized as follows. In section II, the authors go through the operational analysis of a maritime surveillance operation where they particularly focus on the role of the tactical coordinator. They also introduce the Medusa project and how the human behavior has been integrated in high-tech maritime surveillance. Section III starts with an insight into the theory of mental representations. Then, the authors describe their approach and propose to use mental representations of tasks, human actors, human roles, knowledge and abilities. The new workload estimation method is presented in section IV. A scenario example, a workload graph and results of the experiments illustrate the authors' approach. Finally, section V draws some conclusions.

\section{OPERATIONAL ANALYSIS}

In system engineering, the Human View (HV) is required to explicitly represent the human and to document the unique implications humans bring to the system design. It provides a way to integrate human system into the mainstream acquisition and system engineering process by promoting early and frequent consideration of human roles. The purpose of a $\mathrm{HV}$ is to capture the human requirements and to inform on how humans interact with systems. The NATO Architecture Framework (AF) which builds on the United States Department of Defense AF (DoDAF) and the United Kingdom Ministry of Defence AF (MODAF); is the most extensive and complete HV [13, 14]. The Operational Analysis (OA) is the entry point to the analysis of human activities and constraints, called human integration process. The OA consists in identifying operational requirements from the operational concepts. Scenarios with representative data are strongly recommended to support the identification of the dynamic aspects of the human interaction with the system. They are mandatory to be able to apply most of the metrics. The human activities are identified in the Operational View (OV) and the $\mathrm{HV}$ is produced as follows. First of all, activities are refined to produce a task model. The tasks are enriched with data and Knowledge, Skills and Abilities (KSA) requirements. Then, the human roles are identified in the OV and the KSA requirements for the roles can be inferred from the tasks. In a multi-user case, the role structure is also determined. After allocating the human roles to human entities, the required KSA for these entities are deduced. Next, we describe the operational analysis that has been carried out in the context of the Medusa project.

\section{A. Integrating the human behavior in high-tech maritime surveillance}

At a time when we are witnessing an explosion in publicly available information technology, the development of humancentred rather than machine-centred applications is becoming a priority. The workload of operating surveillance aircraft is considerably increased by the range of on-board sensors: radar, optical and infrared cameras, radar detectors, ultraviolet scanners to detect deliberate pollution, AIS for ship identification and new means of communication for getting rapidly in touch with decision-makers and relevant public authorities. The aim of the Medusa project is to introduce behavioural aspects of user-system interaction upstream in the design of new systems for dealing with maritime emergencies. An iterative methodology will be used to reconcile the need to make a system user-friendly, easy-to-learn and efficient with its complexity and the multiplicity of interactions involved. Intended for use by the French government maritime initiative, Action de l'État en Mer, and for managing maritime shipping, Medusa will enhance operators' responsiveness in stressful situations and will facilitate decision-making.

\section{B. Operational analysis: Medusa case study}

We go through the operational analysis of a maritime surveillance operation and focus particularly on the role of TACtical COordinator (TACCO). We suppose that a unique operator holds this role and thus manages the tactical situation. As shown in Fig. 1, the role of TACCO is composed of four main tasks: produce Forward Looking Infra-Red (FLIR camera) video, produce Radar video, manage track list and track classification. The operator can switch over manage track list or track classification and iterate them as many times as necessary.

Produce Radar video and FLIR video are both subautomatic tasks. The operator has only to switch on/off the Radar and the FLIR camera. To manage the track list, the operator builds a Track Wide Scan (TWS) zone (zone of automatic radar tracking) and updates this list. The operator has also to configure the Radar: configuration of the emitter, the receiver, the antenna, wavelength, scanning strategies, etc. He builds manual tracks or tracks on echoes. When necessary, he decides to delete a track and can set a living track as dead reckoning. For the latter status, the ship's current position can be computed by means of a previously determined position. Also, the current position can be fixed and advanced based upon known or estimated speeds over elapsed time, and course. 
Once he has created a set of tracks within the defined area of surveillance, the operator selects some tracks for classification. Both Radar and FLIR classifications can be performed as many times as necessary for each selected track. In order to compute the boat length, the human actor requests a Dynamic Range Profile (DRP) image or an Inverse Synthetic Aperture Radar (ISAR) image and proposes a Radar classification.

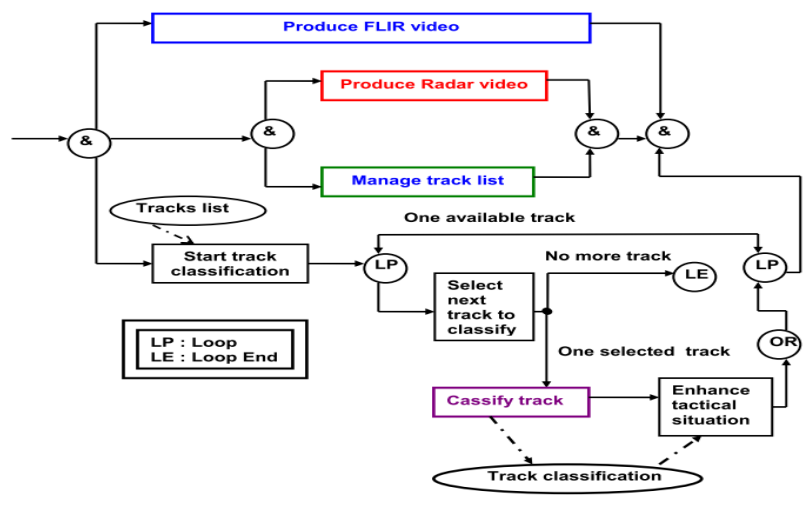

Fig. 1. Manage the tactical situation diagram.

\section{MENTAL REPRESENTATIONS MODEL}

Although the classic models of knowledge representation are not recent, they remain widely used. First, Belief Desire Intention (BDI) architectures [15, 16] describe the internal state of an agent by the mental attitudes of beliefs, goals and intentions. BDI theories provide a conceptual model of the knowledge, the goals and the commitments of an agent.

In the late nineties, Ross Quillian introduced semantic networks as a method of modeling the structure and storage of human knowledge in the shape of a graph [17]. Quillian wanted his system to explore the meaning of English words by the relationships between them. In particular Quillian's system sought to compare words and express the results of those comparisons. Thus, a semantic network is a structure of directed graph, without any circuit, which encodes the taxonomic knowledge by objects as well as their properties by a double labeling. The nodes represent concepts or words that the system "knows" about. Each arc between two nodes represents a semantic relation between two concepts such as the "is-a" relationship, a modification (adjective or adverb), a conjunction (and), a disjunction (or), similarity, consequence, etc. In this way, the representation of the knowledge (of common sense) is simpler and more natural than with the predicate logic. Adding to that, the ease of search for information necessary for certain reasoning and inferences, explains also the popularity of semantic networks. Nevertheless, their semantics remains vague: quantization problems; the transcription of a sentence in semantic network is a delicate problem, since there is no unique (universal) solution. Another defect in this model is that it is not planned to represent correctly formal semantics such as the inference. Even though relations of inference are used for the inheritance, semantic networks do not admit a correct transitivity.

However, there are two derived models with less vague and more formal semantics. They are the conceptual graphs (Sowa,
1976) [18] and the description logic [19]. Finally, neural networks started in 1943 by the presentation of McCulloch and Pitts [20] about the formal neuron which is an abstraction of the physiological neuron. Neural networks yet require too much processing to be functional.

As exposed above, the formalization of knowledge can rapidly become very complex. In our analysis, we propose to use mental representations.

\section{A. Mental representations: structure and operations}

The Theory of Mental Representation (TMR) (Reboul \& Moeschler 1998) [21] aims to complete the Relevance Theory (Sperber \& Wilson 1986) [22] on the specific issue of enrichment of logical form where reference assignment is concerned. It postulates that reference assignment is never entirely done at the linguistic level and makes the strong hypothesis that reference assignment goes through Mental Representations (MRs). MRs are structured representations which gather heterogeneous information, visual, spatial, linguistic and encyclopedic. Their composition includes: an address which is a means of access, a logical entry containing the logical relations between the concept and other concepts, an encyclopedic entry containing both information inherited by default from the generic concept to which the object concerned belongs and information specific to the object, a visual entry including information relative to the present and past appearance of the object, a spatial entry for the intrinsic orientation of the object, its spatial relations to other objects in the same space and its movements, a lexical entry representing the counterparts of the concept in natural languages; i.e., linguistic expressions used to refer to the object and their possible morphological derivations. The visual and spatial entries are addition to the composition of concepts given in Relevance Theory; since operations on MRs can be triggered by perception as well as by discourse. The operations on MRs are the following: creation, modification, fusion, duplication, grouping and extraction.

There are different types of MRs organized hierarchically according to Fig. 2. Any MR inherits from one of the two basic MRs-parents: object and eventuality. The starting point of Eventuality MRs is the ontology of eventualities proposed by Vendler (1957) [23] who distinguished between two major types of eventualities: states and events. In fact, there are two main reasons to represent events in TMR. First, events can be designated by referring expressions (the classification of the track, the configuration of the Radar, etc.) and, given that a basic principle in TMR is that referring expressions are resolved on MRs, events must have MRs corresponding to them. Furthermore, objects can be designated through present or past states (the deleted track/the track that was classified) and these states are the consequence of various events. Finally, events are subdivided into three categories: accomplishments, achievements and activities. According to Vendler, activities and accomplishments are distinguished from achievements in that the former allow the use of continuous and progressive aspects. Activities and accomplishments are distinguished from each other by boundedness: activities do not have a terminal point (a point before which the activity cannot be said to have 
taken place, and after which the activity cannot continue - for example "The TACCO drew a TWS zone") whereas accomplishments do. Of achievements and accomplishments, achievements are instantaneous and take place immediately (such as in "recognize" or "find") whereas accomplishments approach an endpoint incrementally (as in "classify a track" or "adjust the FLIR parameters").

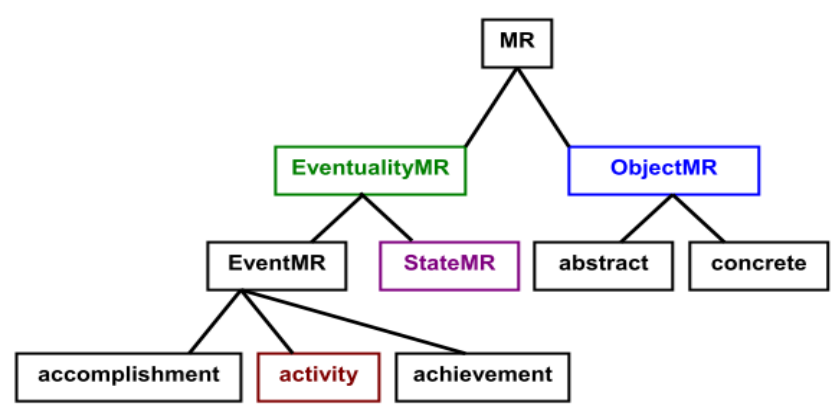

Fig. 2. Hierarchy of mental representations and ontology of eventualities.

\section{B. Mental representation model for the role of TACCO}

For the Medusa project, we are particularly interested in four types of MRs: objects (concrete or abstract), eventualities, states and activities. The subsections below represent our mental model of human entities, human roles, tasks, knowledge and abilities for a maritime surveillance operation, the target application in Medusa. We illustrate our models with examples using graphical representations of MRs.

\section{1) Mental representation of human actors and roles}

In a maritime surveillance operation, roles are generally distinct from human actors. On the one hand, an operator can perform several roles. On the other side, a given role can be distributed across several operators. For example, let us suppose that the surveillance maritime (SurMar) crew is composed of five operators in total representing the cockpit crew and the cabin crew. Piloting the aircraft is the unique role assigned to the pilot; whereas piloting is part of the co-pilot's responsibilities who commands also the aircraft. The role of observing is shared between two different operators. Finally, mission command and sensor management are allocated to the radarist operator who elaborates the tactical situation.

We propose to model a human entity using a concrete, animate and human object concept. Fig. 3 shows the corresponding MR-Object internal structure. In the conceptual entry, operator[1] refers to the category and the cardinal. The notation field contains four properties. In fact, we characterize an operator by his first name, his last name, the knowledge he has acquired and the abilities he has developed.

Furthermore, we model the role of TACCO using an abstract object concept. As for the operator, the properties can be found in the notation. In fact, some knowledge and abilities are required to perform the role of TACCO. Furthermore, a role has a complexity computed in terms of number of MRs, as well as a temporal structure. The temporal structure is a complex propositional content corresponding to the application of a loop (iteration) or binary operators (such as conjunction, disjunction and conditional clause) to one or several propositions. Thus, the temporal structure is like a detailed graph grouping all the possible executions of the allocated tasks.

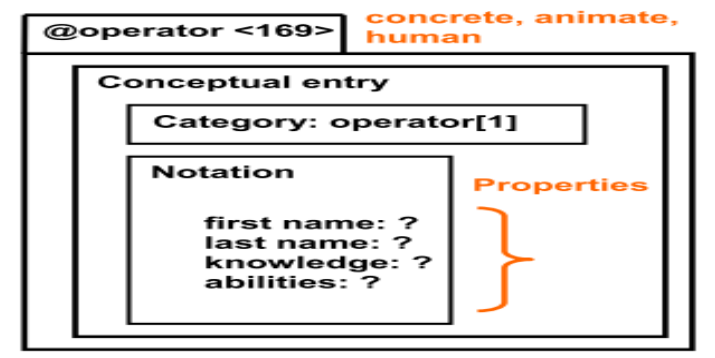

Fig. 3. Object mental representations: internal structure of an operator.

Since the role is the execution of many tasks over time, we have defined a partition of four possible tasks, already presented in section II (see Fig. 1). This partition is available in the logical entry of the MR object. The next paragraph deals with task model.

\section{2) Mental representation of tasks}

We propose to model tasks using events MRs. Thus, a task can be an accomplishment, an activity or an achievement. We also decompose each task into subtasks. For instance, the task "perform FLIR classification" consists of three subtasks: get ship FLIR image, decorate FLIR image and propose FLIR classification. As usual, we refer to the category and the cardinal in the conceptual entry. In the notation, we implement the same properties already defined for the role of TACCO: required knowledge, required abilities, temporal structure and complexity. Adding to that, an event has a temporal location and a begin time that can be found in the field Time of the conceptual entry.

We apply the mental model to manage track list. The task is divided into eight subtasks: create a TWS zone, configure the Radar, build/ delete a track, automatic/manual tracking, set living track as dead reckoning and end track list update. This partition is represented in the logical entry of the parent MR, where the parts can be differentiated according to their generic concept and the begin time of the corresponding event. The temporal structure of this task can be described as follows: $\left\{\mathrm{P}_{1}\right.$ $\left.\left.\&\left\{\mathrm{LP}\left(\left\{\mathrm{P} 2 \||| \mathrm{P}_{3} \mathrm{~V} \mathrm{P} \mathrm{P}_{4} \mathrm{~V} \mathrm{P}_{5} \mathrm{~V} \mathrm{P}_{6} \mathrm{~V} \mathrm{P}_{7} \mathrm{~V} \mathrm{P}_{8} \mathrm{~V} \mathrm{P} \mathrm{P}_{9}\right\}\right\}\right)\right\}\right\}$; where:

$$
\begin{aligned}
& \mathrm{P}_{1}=@ \text { create_zone }<25>, \mathrm{P}_{2}=@ \text { exploit_Radar_video }<62>, \\
& \mathrm{P}_{3}=@ \text { radar_menu }<33>, \mathrm{P}_{4}=@ \text { build_track }<20>, \\
& \mathrm{P}_{5}=@ \text { delete_track }<28>, \mathrm{P}_{6}=@ \text { automatic_tracking }<40>, \\
& \mathrm{P}_{7}=@ \text { manual_tracking }<41>, \mathrm{P}_{8}=@ \text { dead_reckoning }<63>, \\
& \mathrm{P}_{9}=\text { @end_track_list_update }<64>,
\end{aligned}
$$

LP represents an iterative process, $\mathrm{V}$ is a disjunction, \& is a conjunction and III shows that subtask "exploit Radar video" is simultaneously executed with all the remaining subtasks (parallelism).

During a maritime surveillance operation, we suppose that the TACCO operator first created a TWS zone. Then, he configured the Radar and created two tracks. He performed automatic tracking for the former and manual tracking for the 
latter. Finally, he ended his tracks list update. The temporal structure is a complex proposition representing the conjunction of seven propositions as follows: create_zone $<256>\&$ $\{$ @exploit_Radar_video $<261>$ III $\quad$ radar_menu $<257>\quad \&$ build_track $<258>\quad \& \quad$ build_track $<259>\quad \&$ automatic_tracking $<260>\quad \& \quad$ manual_tracking $<261>\quad \&$ end_track_list_update $<260>$ \} \} .

As far as task "perform FLIR classification", the temporal structure is the conjunction of three propositions corresponding to the aforesaid subtasks: \{@get_boat_FLIR_image<84> \& $@$ decorate_FLIR_image $<85>$ @ propose_FLIR_classification $<86>$ \}.

Fig. 4 is another application of the task model in the case of camera_on, one of produce_flir_video subtasks. In order to switch on the FLIR camera, a human actor acts on the camera concept, and this object participant is defined in the conceptual entry of camera_on (argD). The required knowledge and abilities are also compulsory to evaluate the complexity of a task. Below, we address these issues in details and we introduce the mental model we have adopted for knowledge and abilities.

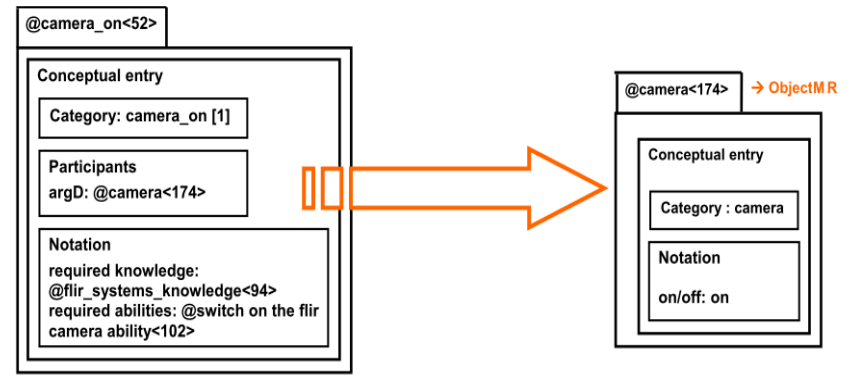

Fig. 4. Event mental representation: internal structures of the subtask camera_on.

\section{3) Mental representation of knowledge and abilities}

As exposed at the beginning of section III, the formalization of knowledge and abilities can rapidly become very complex. However, we need a simple formalism that enables matching between roles and human actors. Knowledge designates the familiarity with information, facts and descriptions. Abilities designate aptitude and intelligence. They are competences to perform an activity. We do not include skills in the model since we consider them as reflex behaviors. For these reasons, we consider knowledge and abilities as mental states and we model them by state MRs, as shown in Fig. 5.

The knowledge model has two participants and one property: agent, know and level, respectively. The agent participant refers to the operator executing the current task. For example, an operator must acquire radar_systems knowledge to be able to configure the Radar. The required level for this knowledge is $\mathrm{C}$ (proficient) since this configuration includes the emitter, the receiver, the antenna, the wavelength, the scanning strategies, etc.

We propose a similar structure to model abilities: two participants (agent and know how to) and one property (required expertise level). The expertise level property takes the values of novice, advanced beginner, competent, proficient or expert, inspired from the 'novice to expert' Dreyfus model [24]. For example, an operator can perform a Radar classification if he has developed the corresponding ability with the highest expertise level (i.e., expert).
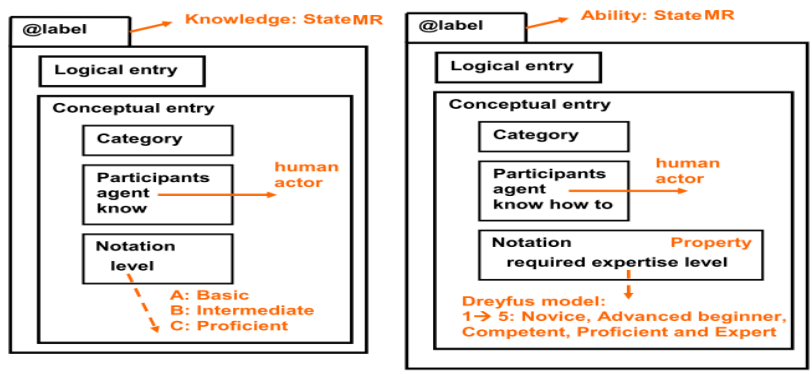

Fig. 5. State mental representation: generic internal structure of knowledge and abilities.

To make sure a given role goes smoothly, it is necessary to check that the human actor has acquired some concepts and can put his abilities to the best use. The role allocated to the operator will hopefully go off to a good end. Thus, roles and entities matching is a two-folded matching:

- Matching the required knowledge and the operator acquired knowledge;

- Matching the minimum required and the operator acquired expertise level for all the abilities.

In fact, to carry out the role of TACCO, it is necessary to have acquired the following knowledge: identification systems (AIS, IFF), radar systems (radar, ISAR, TWS, DRP, SAR and ISAR library), FLIR systems (FLIR, FLIR library), maritime knowledge (maritime environment), meteorology, communications knowledge (navigation systems, GPS), etc. The level of knowledge differs from one task to the other. For example, a B level (intermediate) maritime knowledge is enough to manage_track_list. However, performing track classification requires a C level (proficient) maritime knowledge. Several workshops and interviews were held with TACCO operators in order to accurately estimate subjective factors such as the level of experience and the required expertise level.

We have developed a graphical interface to instantiate an operator and match his knowledge and abilities with those required to carry out the role of TACCO. In the sequel, we suppose that the matching goes off smoothly and we attribute the role of TACCO to a convenient operator. It is possible to observe him in situation, estimate the mission completion time, estimate and analyze his workload. Applied metrics allow to verify the capacity of the human actor to perform the tasks he has been allocated. Section IV focuses on workload issues.

\section{TOWARDS AN ESTIMATION OF THE WORKLOAD}

We propose to estimate the workload $W$ of a maritime surveillance operator, in terms of number of MRs, by means of the formula $W=\sum_{i=1}^{n_{t}} \sum_{j=1}^{T L_{i}}\left(1-\rho_{j}\right) \times C_{j}(M R s)$, where: 
- $n_{t}$ is the number of tasks considered within a given role;

- Factor $\rho_{j}$ is linked to the operator's experience. In fact, the more experienced the operator is, the less complex the task is and the less load over time it requires;

- Task complexity $C_{j}$ depends on both the level of required knowledge for the task (A, B or $\mathrm{C}$ ) and the required expertise levels of the different abilities (novice, advanced beginner, competent, proficient, expert). The task complexity remains unchanged if the expertise level is "novice" or "advanced beginner". However, it is doubled for a "competent" required level, tripled for "proficient" and quadrupled for "expert". According to the knowledge level, the complexity is doubled for A-level (basic), tripled for B-level (intermediate) and multiplied by four for Clevel (proficient).

- Factor $T L_{i}$ represents the load over time; and is computed according to the temporal structure of the task.

It is important to differentiate $T L$ from $C$. Although subtasks "build fifty tracks during two hours" and "build fifty tracks in five minutes" have the same complexity, they have different loads over time.

Let us consider produce_Radar_video task for example. The corresponding event MR contains a partition of four subtasks in the logical entry: radar_on, provide_drp, provide_isar and radar_off. A differentiation criterion, based on the category and the begin time of the subtask, is used to isolate each part from the others. The temporal structure is the conjunction of four propositions corresponding to the aforesaid subtasks; and it is expressed as follows: \{ @ radar_on $<58>\&$ @ provide_drp $<60>\&$ @ provide_isar $<61>\&$ @ radar_off $<59>$ \}. Thus, the time load $T L$ of produce_Radar_video task is computed by means of the complex proposition above: $T L=4$. As far as task complexity is concerned, we analyze the required knowledge and abilities. For radar_on and radar_off subtasks, the operator should know the concept of radar. As only Alevel (basic level) knowledge of this concept is required, the complexity is then doubled. The corresponding object MR ( $\operatorname{argD}$ participant) has a property on/off which is also updated. Furthermore, an advanced beginner is the minimum threshold identified as required expertise level to switch on/off the Radar. With this property value, the complexity of the subtask remains unchanged. Then for radar_on and radar_off the complexity is: $C=1+2+1+1=5 \mathrm{MRs}$, corresponding to the MRs of the event itself, A-level, advanced beginner and $\operatorname{argD}$ participant values. Similar reasoning is applied for subtasks provide_isar and provide_drp. The operator should have acquired A-level knowledge of the concepts isar and $d r p$ respectively. The $\operatorname{argD}$ participants are also updated and there is not any ability for these automatic subtasks. In this case, $C=1+2+1=4 \mathrm{MRs}$, which corresponds to the MRs of the event itself, A-level and argD participant values. As produce_Radar_video is a subautomatic task, it does not need an expert to be accomplished. For this reason, we consider factor $\rho=0$, since the experience and training of the operator slightly influence the workload in the example. Finally, the estimated workload for produce_Radar_video is $W=2 \times 5+2 \times 4=18$ (MRs).

Let us assume that we have observed a maritime surveillance operator on Dassault's Falcon 50 aircraft. Fig. 6 represents the graphical interface used to simulate the execution for the role of TACCO. Below is a selection among the tasks he has accomplished during the mission. First of all, the operator switched on the FLIR and the Radar. Then, he configured the Radar. He exploited the displayed video and drew a TWS zone. Then, he built five tracks. He decided to delete one of them. After that, he classified one track based on a FLIR classification. A Radar and FLIR classifications were necessary to classify the second track. The operator classified the third track after two Radar image-based classifications (ISAR and DRP). Finally, he updated the tracks list by setting a living track as dead reckoning.

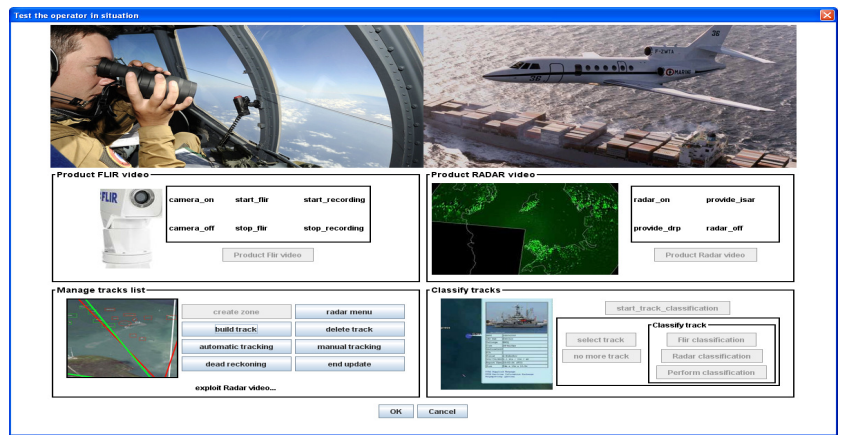

Fig. 6. Role execution via the graphical interface: a scenario example.

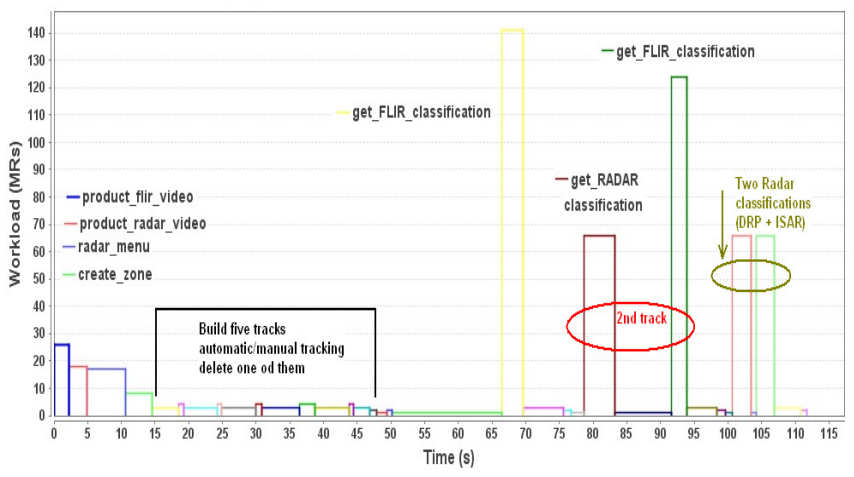

Fig. 7. Workload graph: execution via the graphical interface.

The workload graph provided by Fig. 7 shows some peaks. In fact, even for a well trained and experienced operator, track classification remains the most demanding task. There are mainly five peaks: two of them correspond to FLIR classifications (141 MRs and $124 \mathrm{MRs}$ ). The three others of 66 MRs belong to Radar classification subtasks. These were the most complex tasks throughout the previous scenario. At the beginning, the operator configured the Radar. The workload necessary to carry out this task is about 17 MRs. Note that, even though building tracks does not represent a complex task, its repetition entails a significant time load.

A series of experiments were conducted at Thales (Brest, France) with a maritime surveillance crew in December 2013 
and February 2014. The radar operator was equipped with several sensors -such as a contactless eye tracker, an electrocardiogram (ECG)-enabled armband, a wireless heart rate monitor, etc - to measure the psycho-physiological signals. Fig. 8 shows a twenty-minute portion of the raw ECG recorded during the experiments. We have associated the corresponding tasks executed by the radar operator. We notice that the high peaks correspond to demanding tasks such as tracks classification or tactical situation enhancement. Whereas, a consequent reduction of the workload corresponds to simple tasks such as displaying the cartography, zooming in/out, creating or deleting tracks, etc. Therefore, the ECG is coherent with the workload graph generated by the model, as it was similarly observed in [25].

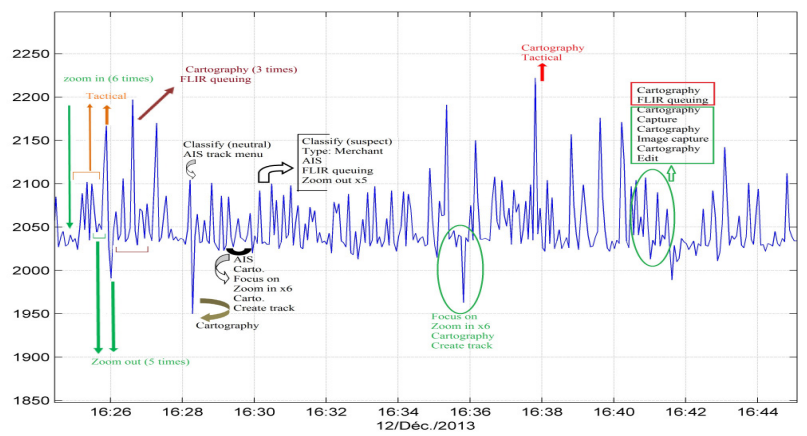

Fig. 8. A sample of the recorded ECG of the radar operator.

\section{CONCLUSION}

In this paper, we have proposed a new method to measure the workload based on three important parameters: the experience and training of the human actor, the complexity of the task and the time load. Our model is inferred from tasks analysis. We have proposed mental representations of human entities, human roles, tasks, knowledge and abilities. The required knowledge and abilities for each task affect the corresponding complexity. In fact, the higher the required expertise level is, the more complex the task is. We have investigated the mental representations as well as workload issues to model a maritime surveillance operation, particularly for the role of TACCO. Finally, experiments with maritime surveillance operators were carried out to give concrete expression to the predictive workload estimation and validate the proposed analysis.

The approach proposed is generic and is currently being applied to another use-case (airborne SIGnal INTelligence SIGINT) for further validation. In addition, the model is being refined based on some interviews we conducted with TACCO operators. The main perspective consists in adding the influence of the physical environment and the physiological constraints.

\section{REFERENCES}

[1] Mulder, G.: Mental load, mental effort and attention. Mental Workload. Its Theory and Measurement, 299--326 (1979)

[2] Colle, H., Reid, G.: Double trade-off curves with different cognitive processing combinations: Testing the cancellation axiom of mental workload measurement theory. Human Factors: The Journal of the Human Factors and Ergonomics Society, SAGE Publications, 41, 35--50 (1999)

[3] Sirevaag, E., Kramer, A., Wickens, C., Reiswerber, W., Stayer, D., Grenell, J.: Assessment of pilot performance and mental workload in rotary wing aircraft. Ergonomics, Taylor \& Francis, 36, 1121--1140 (1993)

[4] Yeh, Y., Wickens, C.: Dissociation of performance and subjective measures of workload. Human Factors: The Journal of the Human Factors and Ergonomics Society, SAGE Publications, 30, 111--120 (1988)

[5] Johannsen, G., Moray, N., Pew, R., Rasmussen, J., Sanders, A., Wickens, C.: Final report of experimental psychology group. Mental workload: Its theory and measurement, New York: Plenum, 101--114 (1979)

[6] Muckler, F., Seven, S.: Selecting performance measures:" Objective" versus" subjective" measurement. Human Factors: The Journal of the Human Factors and Ergonomics Society, SAGE Publications, 34, 441-455 (1992)

[7] Tattersall, A., Foord, P.: An experimental evaluation of instantaneous self-assessment as a measure of workload. Ergonomics, Taylor \& Francis, 39, 740--748 (1996)

[8] Hart, S., Staveland, L.: Development of NASA-TLX (Task Load Index): Results of empirical and theoretical research. Human mental workload, Amsterdam, Holland, 1, 139--183 (1988)

[9] Pfendler, C., Widdel, H.: Gedächtnisleistung und Beanspruchung beim Wiedererkennen von farbigen und schwarzen Reizmustern auf elektronischen Anzeigen. FAT Bericht (1988)

[10] Sepehr, M.: Assessment of subjective mental workload using NASATask load index. Proceedings of the 7th European Annual Conference on Human Decision Making and Manual Control, 69--75 (1988)

[11] Veltman, J., Gaillard, A.: Indices of mental workload in a complex task environment. Neuropsychobiology, Karger Publishers, 28, 72--75 (1993)

[12] Veltman, J., Gaillard, A.: Measurement of pilot workload with subjective and physiological techniques. In Proceedings of the Workload Assessment and Aviation Safety Conference (1993)

[13] The NATO Human View Handbook

[14] NATO Human View Quick Start Guide

[15] Bratman, M., Israel, D., Pollack, M.: Toward an architecture for resource-bounded agents. SRI and Stanford Univ., Stanford, CA, Centre for the Study of Language Information, CSLI-87-104 (1987)

[16] Rao, A., Georgeff, M.: Modeling Agents Within a BDI-Architecture. In R. Fikes and E. Sandewall, editors, Proc. of the 2rd International Conference on Principles of Knowledge Representation and Reasoning (KR'91), Cambridge, Mass, 473--484 (1991)

[17] Quillian, M. R.: Semantic memory. In M. Minsky, (Ed.), Semantic information processing. Cambridge, MA: MIT Press, 216-270 (1968)

[18] Sowa, John F.: Conceptual graphs for a database interface. IBM Journal of Research and Development, 20:4, 336--357(1976)

[19] Brachman, R., Schmolze, J.: An overview of the KL-ONE knowledge representation system. Cognitive science, Elsevier, 9, 171--216 (1985)

[20] McCulloch, W., Pitts, W.: A logical calculus of the ideas immanent in nervous activity. Bulletin of mathematical biology, Springer, 5, 115-133 (1943)

[21] Reboul, A., Moeschler, J.: Pragmatique du discours. De l'interprétation de l'énoncé à l'interprétation du discours (1998)

[22] Sperber, D., Wilson, D.: Relevance: Communication and cognition. Blackwell, Oxford, 142 (1986)

[23] Vendler, Z.: Verbs and times. The philosophical review, JSTOR, 143-160 (1957)

[24] Dreyfus, S.E. and Dreyfus, H.L.: A five-stage model of the mental activities involved in directed skill acquisition. DTIC Document (1980).

[25] Kbaier Ben Ismail, D. and Grisvard, O. : A model-driven approach to the a priori estimation of operator workload. IEEE International MultiDisciplinary Conference on Cognitive Methods in Situation Awareness and Decision Support (CogSIMA), March 9-12, Orlando, USA (2015) 